\title{
CONOCIMIENTO DE LAS PERSONAS ADULTAS SOBRE EL CLIMATERIO, ANDROPAUSIA Y LA SEXUALIDAD
}

\section{KNOWLEDGE OF ADULTS ABOUT THE CLIMACTERIC, ANDROPAUSE AND SEXUALITY}

\author{
Viriam Leiva Díaz \\ Carolina Arguedas Medino ** \\ Marilyn Hidalgo Castillo \\ Yessenia Navarro Vargas ${ }^{* * * * *}$
}

RESUMEN

\begin{abstract}
El presente artículo expone los resultados de una investigación, la cual tuvo como objetivo principal diagnosticar el conocimiento y vivencias que poseen las personas adultas, con respecto al proceso de climaterio y la sexualidad. Este estudio tuvo un enfoque cuantitativo con un diseño metodológico de tipo estructural descriptivo. La información se obtuvo por medio de un cuestionario aplicado a una muestra de 176 personas, de las cuales 108 eran mujeres y 68 varones, con edades que oscilaban entre los 35 a 65 años. Entre los resultados más destacados se encontró que la población posee vacíos de información en los temas de sexualidad y sus componentes. Se demostró que la mayoría manejan conceptos erróneos en cuanto a la temática, además de tabúes y mitos.
\end{abstract}

PALABRAS CLAVE: CLIMATERIO * SEXUALIDAD * EROTISMO * GÉNERO * SEXO * AUTOESTIMA

$*$ RELACIONES INTERPERSONALES * ESPIRITUALIDAD

Escuela de Enfermería de la Universidad de Costa Rica (UCR).

viriaml@gmail.com

** EBAIS del Hospital Clínica Bíblica, Costa Rica

caroarme@gmail.com

**** Hospital Calderón Guardia, Costa Rica.

laflaca1099@hotmail.com

**** $\quad$ Empresa Volcano, Costa Rica.

yesse.n.v@gmail.com 


\section{ABSTRACT}

This article exposed the results of an investigation about the knowledge that adult people have of the climacteric's process and the sexuality. This study had a quantitative approach with a methodological design of structural descriptive type. The information was obtained by questionnaire. It was applied to a sample of 176 people (108 women and 68 men) with ages between 35 to 65 years old. The most important results were the information gaps in the topics of sexuality and its components. Most people have wrong knowledge in this topic, also many taboos and myths.

KEYWORDS: CLIMACTERIC * SEXUALITY * EROTICISM * GENDER * SELF ESTEEM * INTERPERSONAL RELATIONSHIPS * SPIRITUALITY

\section{INTRODUCCIÓN}

El ser humano durante el ciclo vital de su desarrollo, debe enfrentar y superar diferentes etapas, las cuales son inminentes en el transcurso de su vida. Las mismas generan cambios en múltiples aspectos, como por ejemplo, durante la vivencia de su sexualidad; asimismo, algunas de estas fases corresponden a la etapa del climaterio y la andropausia (Dulcey, 2000).

En estas etapas se presenta una serie de cambios en los sujetos que transitan por este período, los cuales pueden modificar la vida cotidiana y la vivencia personal, lo que significa muchas veces, el desencadenamiento de una crisis; sin embargo, es importante tener en cuenta que esta realidad es diferente en cada sujeto.

Aunado a lo anterior, cabe subrayar que cada individuo posee diferentes formas para enfrentar estos nuevos procesos, dependiendo de las experiencias de vida $y$ del entorno en el que se ha desenvuelto la persona, así como, el conocimiento que posee sobre esta etapa que atraviesa $y$ la forma en que ha logrado superar, con éxito o no, las anteriores etapas del desarrollo.

Todos los seres humanos viven su sexualidad desde sus particularidades, por lo cual es significativo destacar la importancia que posee la vivencia de la misma para el ser humano, ya que forma parte de su desarrollo integral.

Para las personas es indispensable conocer, vivir y expresar su sexualidad, independientemente de sus características personales, ya que esta permite transcender a un ámbito más espiritual y psico-emocional, en la cual se da la expresión más perfecta de los sentimientos, las emociones, los valores y el amor entre otros. Además, incluye las relaciones que tenemos con los demás y con nosotros mismos; es por esto que a través de la sexualidad, las personas sienten, expresan $y$ viven.

A pesar de que el climaterio y la andropausia son etapas inherentes al desarrollo, se da de manera frecuente un desconocimiento de la misma, generando mitos, tabúes y prácticas inadecuadas. Todas estas realidades son comunes en el diario vivir y es importante que las personas construyan estrategias adecuadas para afrontarlas, por lo que es indispensable la colaboración de un profesional que le muestre herramientas necesarias para enfrentar estos estadios, con el suficiente empoderamiento.

\section{METODOLOGÍA}

La investigación tuvo un enfoque cuantitativo con un diseño estructural descriptivo de tipo transversal (Barrantes, 2006). La población estuvo conformada por 1150 asociados y asociadas activos de CooproNaranjo RL, quienes residen en el cantón de Naranjo. Para el estudio se extrajo a través de un muestreo probabilístico por cuotas, una muestra de 176 personas, de las cuales 108 eran mujeres y 68 eran varones, cuyas edades oscilaban entre los 35 y 65 años de edad.

Para la recolección de los datos se utilizó un cuestionario constituido por 27 preguntas. De estas 25 eran preguntas cerradas y trataban sobre los temas de climaterio, sus síntomas y sus signos, asimismo, sobre el autoestima y los componentes de la sexualidad; las otras dos preguntas eran abiertas y correspondían a la 
temática de autoestima y proyecto de vida. Este instrumento se sometió a revisión por parte de cuatro expertos en la temática y en estadística.

Para reunir la información, se procedió a asistir a cada casa de los asociados y asociadas, para ello las personas procedieron a llenar el consentimiento, el cual se diseño basándose en el documento que posee la Vicerrectoría de Investigación de la Universidad de Costa Rica.

La duración del estudio fue de cuatro meses (de junio a octubre de 2010), desarrollado en el cantón de Naranjo de la provincia de Alajuela.

Asimismo, toda investigación que tenga como objeto de estudio al ser humano, debe regirse por el conjunto de principios éticos generales, establecidos por las regulaciones nacionales e internacionales, teniendo como norte fundamental los derechos humanos, como inviolables $y$ los cuales se encuentran por encima de los intereses de cualquier investigación (Chacín y Briceño, 2001).

Entre los criterios de Bioética considerados en esta investigación se encuentran: el "principio de respeto", pues se aplicó un consentimiento informado, en el cual se les explicó a los y las participantes el propósito del trabajo; "el principio de beneficencia" donde se realizó una aclaración sobre los riesgos, beneficios y consecuencias de participar en la investigación; así como, se les aseguró a los participantes confidencialidad en la información brindada, pero se les informó que los datos serían divulgados en una publicación científica. Por último, se consideró el "principio de justicia", dando un trato igualitario a cada participante durante el proceso.

\section{RESULTADOS}

Como se mencionó previamente, la muestra estuvo constituida por 176 participantes de los cuales, 108 eran mujeres y 68 varones, en edades que oscilaban entre los 35 y 65 años.

Dentro de los aspectos sociodemográficos importantes para el estudio se encuentra el estado civil, 153 de las personas participantes son casados(as), 19 son solteros(as), 9 se encuentran divorciados(as), 3 son viudos(as) y 2 personas vivían en unión libre. Además en cuanto a la convivencia familiar,
156 personas viven con su esposo(a), 134 viven con sus hijos(as), 6 viven con los padres, 4 con los suegros(as) y 4 viven con otras personas.

Entre las ocupaciones laborales que desempeñaban los entrevistados(as), se encontró que $29 \%$ son agricultores, un $57 \%$ son amas de casa, otro $3 \%$ son maestros(as) y el $19 \%$ restante practica otro oficio (cocineros, misceláneos, ingenieros, arquitectos, enfermeras, entre otros).

En lo que respecta a la escolaridad, se obtuvo que 25 personas finalizaron estudios universitarios, seguida por 22 de ellos que no la terminaron; además, 25 personas poseen una secundaria incompleta y 20 poseen secundaria completa; 19 asociados tienen la primaria incompleta, 59 mantienen primaria completa; por otra parte, 6 poseen otro grado de escolaridad y solamente 1 persona, no posee estudios primarios.

Teniendo en cuenta la descripción de las personas entrevistadas, se analizarán los resultados obtenidos sobre los conocimientos y prácticas de las personas en torno a las etapas de climaterio $y$ andropausia.

En cuanto al conocimiento sobre el concepto de climaterio se encontró que un $64 \%$ refirieron conocer el concepto, mientras el restante 36\% mencionó desconocerlo. De los que afirmaron conocer el concepto, solo un $48 \%$ concordaron que es el periodo de transición entre la vida reproductiva y la no reproductiva, que comprende la menopausia y la andropausia. Otro porcentaje representativo del $52 \%$ manejan un concepto erróneo del mismo.

En lo que respecta a la edad que abarca el climaterio, solo un 17\% (30) mencionó correctamente que oscila entre los 35 y 65 años, mientras un $83 \%$ mencionaron que estas etapas iban desde los 25 a los 75 años de edad.

Por otra parte, al indagar sobre cuáles consideraban que eran los cambios que se dan en esta etapa, se evidenció que la mayoría de la población refirió como síntomas presentes: una disminución del deseo sexual, cambios en el estado emocional, sudoración aumentada, retraso en la lubricación vaginal, así como, sensación de bochorno y escalofríos repentinos. A su vez, un porcentaje más pequeño de 
participantes (15\%) manifestaron que también la incapacidad para lograr o para mantener una excitación sexual, los sentimientos de incomprensión de las otras personas hacia la realidad de vida, disminución en la potencia durante la erección, mayor cantidad de tiempo para alcanzar el orgasmo y un cambio en los patrones de sueño.

Unido a lo anterior, se preguntó cuales de los cambios mencionados estaban presentando los y las participantes, encontrándose en primer lugar, una disminución del deseo sexual, cambios en el estado emocional, bochornos y escalofríos repentinos, sudoración aumentada, retraso en la lubricación y cambio en los patrones de sueño. A su vez, los síntomas menos frecuentes entre las personas encuestadas estaban: incapacidad para lograr o para mantener una excitación sexual, sentimientos de incomprensión de las otras personas hacia la realidad de vida en esta etapa, disminución de la potencia durante la erección y una mayor cantidad de tiempo para alcanzar el orgasmo. Por último, un $2 \%$ (4) de los asociados(as) consideraron que no habían vivido ningún cambio.

Otro aspecto explorado fueron los mitos $y$ estereotipos que las personas tienen en torno al climaterio. La mayoría de la población maneja uno o varios mitos en torno a esta temática; entre los mencionados frecuentemente se encuentran los relacionados con la actividad coital, por ejemplo: "no se puede tener relaciones sexuales cóitales con la pareja", "el deseo sexual disminuye después de la menopausia". Otros mitos están asociados con aspectos de ánimo, como que "las mujeres aumentan su estado de enojo", y por último, se encuentran los relacionados con el género, tal como que "los hombres no desarrollan cambios". En el estudio realizado se encontró que el 44\% (78) asociados(as) creen que, tanto el hombre como la mujer sufren cambios en la etapa del climaterio, lo cual es correcto, puesto que ambos lo experimentan.

Por otra parte, al indagar sobre cuáles consideraban que eran los cambios que se dan en esta etapa, se evidenció que la mayoría de la población refirió como síntomas presentes: una disminución del deseo sexual, cambios en el estado emocional, sudoración aumentada, retraso en la lubricación vaginal, así como, sensación de bochorno y escalofríos repentinos. A su vez, en cuanto a las edades que abarcaban la misma, un $74 \%$ de la población manifestó que oscilaba en el intervalo entre 40 a 60 años, un $16 \%$ expresó que tenía lugar entre los 35 y 65 años de edad, y el porcentaje restante (10\%), decidió marcar alguna otra opción diferente.

Al evaluar el concepto de andropausia, la mayoría de la población (61\%) en estudio no conoce qué es o tienen un concepto errado de la misma, solamente un $39 \%$ de todos los encuestados contestaron correctamente al referirse como "la disminución de los niveles de las hormonas masculinas". De igual forma, al indagar sobre las edades que abarcaban dicha etapa de vida, un $54 \%$ lo desconocía, mientras que el $46 \%$ consideró correctamente que la andropausia se desarrolla entre los 40 y 60 años.

Seguidamente, se presentan los resultados obtenidos acerca del conocimiento sobre el término de sexualidad, por lo que es importante aclarar que para el desarrollo de esta temática, se tomaron en cuenta los conceptos que la conforman: sexo, género, erotismo y espiritualidad.

En cuanto al concepto de sexo, solo un $52 \%$ de la población refirió correctamente que es un conjunto de características biológicas que definen al espectro de humanos como hembras y machos, mientras que el otro $48 \%$ mantenía un concepto equivocado sobre la misma o no lo sabían; entre las enunciaciones brindadas están: "construcción social que define a la persona", "relaciones íntimas entre dos personas", entre las más frecuentes.

Por otra parte, en cuanto a la definición de género, se obtuvo que solo el $27 \%$ de las personas concordó con el concepto correcto como aquel conjunto de características sociales y culturales asignadas a las personas en función de su sexo, mientras que un $36 \%$ consideraba que era lo mismo que sexo. El otro $37 \%$ mostró otras concepciones incorrectas o lo desconocían.

$\mathrm{Al}$ indagar sobre el concepto de erotismo, un 35\% acertó al expresarlo como "un conjunto de procesos humanos en torno al apetito por la excitación sexual, la excitación misma y el 
orgasmo, sus resultantes en la calidad placentera de esas vivencias humanas", un $20 \%$ hace referencia a la acciones de ver material pornográfico, y el otro 45\% estableció otros conceptos erróneos o lo desconocían.

Por último, en el concepto de espiritualidad, un $68 \%$ lo destacó como el conjunto de aspiraciones, convicciones, valores y creencias que permiten a cada persona orientar sus proyectos de vida, un $12 \%$ consideró que la espiritualidad se expresa o se vive solamente dentro de una religión y el otro $20 \%$, manejaba otro concepto erróneo de espiritualidad, o no sabía a qué hacía referencia.

\section{DISCUSIÓN}

En este estudio se destaca la participación mayoritaria de mujeres, esto podría estar asociado a que social y culturalmente, los cambios que se gestan en ellas, durante el ciclo de vida estudiado, son referidos con mayor frecuencia. Por otro lado, debido a la socialización masculina, a los varones se les dificulta aceptar la enfermedad, así como, la expresión de sus temores y necesidades, haciéndoles sentir que deben soportar con valentía, sin quejas y en soledad junto a los cambios físicos y fisiológicos que se van dando (Moyniham, 1998 y ops, 2000).

Un aspecto que se indagó fue las redes de apoyo con que cuentan las personas participantes del estudio, esto es fundamental ya que la forma en que se viven los cambios generados en la premenopausia, menopausia, climaterio femenino $y$ andropausia, depende de la posibilidad de contar con redes de apoyo (Montes de Oca, Guzmán y Huenchuan, 2003), con el fin de compartir sus experiencias de vida, contando con apoyo emocional, siendo especialmente importante cuando su compañero(a) está viviendo el mismo proceso, haciendo más fácil la convivencia con estos cambios y por ende, un envejecimiento más saludable (Sánchez, Honrubia y Chacón, 2005). Esto guiaría procesos de educación en salud dirigido a parejas que están atravesando este período de vida.

Por otra parte, el conocimiento sobre la convivencia familiar es otro aspecto relevante, ya que es un factor determinante en cómo los y las participantes viven esta etapa de vida, debido a que la influencia de la pareja y la convivencia en su hogar, influyen en la manera de afrontar los cambios. Según Clavijo (2009), el entorno familiar interviene en forma muy significativa, debido a que estas etapas coinciden con situaciones de crisis, en el interior de la familia, con la pareja, los hijos $y$ los padres, lo que pone en riesgo la estabilidad y el equilibrio logrado por la familia, en etapas previas al climaterio.

Otro aspecto importante que se debe analizar corresponde a los oficios que desempeñan estas personas, ya que esto va a determinar las posibilidades de acceso a los servicios de salud, en el momento que empiezan a presentar los cambios en su cuerpo, así como, poder realizar consultas médicas cuando surjan dudas al respecto (Programa Estado de la Nación, 2009). Unido a esto se encuentra el grado de escolaridad, ya que es otro factor que se debe tomar en cuenta, puesto que el desenvolvimiento académico que exhiban las personas, puede influir en la forma en que ellos viven su sexualidad en estas etapas de vida y en cómo atraviesen los cambios vividos en las mismas. El grado de escolaridad puede ser un factor determinante en el acceso a la información y a su vez, en la búsqueda de ayuda por parte de personas expertas en el tema (Organización Mundial de la Salud, 2010). Asimismo, puede influenciar en el empoderamiento que tengan las personas, en cuanto a su vida $y$ en los efectos de los cambios presentados en estas etapas.

En el tema sobre el conocimiento que poseían los y las entrevistadas acerca de la conceptualización del término de climaterio, se evidenció que el concepto no está claro, aun siendo este un proceso que forma parte del ciclo vital y un período de transición, entre la vida reproductiva y la no reproductiva, hasta la vejez (Medina, 2008).

Ejemplo de lo mencionado previamente se encuentran los resultados en lo que respecta al conocimiento de la edad de inicio de las etapas. De acuerdo con Benítez (2008), el climaterio inicia a los 35 años con el climaterio temprano; a los 45 años, continua con el perimenopáusico y a los 55 años se da el climaterio tardío, el cual terminaría a los 65 años. 
En el caso de los hombres, la andropausia comienza alrededor de los 50 años de edad (Rodríguez, 2009).

El reconocimiento de las diferentes manifestaciones que pueden presentarse en ese proceso, contribuye a disminuir la ansiedad que pueda derivarse de estos cambios, brindándole la oportunidad de entender el proceso, ya que es normal que en la etapa del climaterio, tanto en hombres como mujeres, se den una serie de cambios fisiológicos y emocionales. Como lo mencionan Araya, Urrutia y Cabieses (2006), en el caso de las mujeres se presentan síntomas como bochornos, diaforesis, insomnio, cambios de humor, disminución de la libido, con una alteración en la lubricación vaginal, en el momento del coito.

Del mismo modo, como lo menciona Rodríguez (2009), los cambios en el hombre son variados y están muy relacionados con los que presentan las mujeres; entre ellos se encuentran: una pérdida de la energía y/o la motivación, con disminución de la actividad intelectual y de la memoria. Es común la presencia de fatiga, depresión, cambios emocionales, irritabilidad y debilidad muscular. Asimismo, en la parte sexual se puede encontrar una disminución del deseo, dificultad en lograr y mantener una erección, disminución de erecciones nocturnas y de satisfacción sexual. Además, se observa la reducción de la masa muscular corporal, vigor físico y densidad ósea, dolores osteoarticulares y disminución de la estatura, así como, el aumento del riesgo de padecer osteoporosis. Muchos de estos cambios, descritos por los y las participantes, van en relación con la manera en cómo están atravesando estas etapas, debido a que no todos han presentado la mayoría de estos síntomas, por lo tanto, cada participante lo ve de manera distinta.

Como lo expone Bonifacio et ál., los “(...) síntomas biológicos y psicológicos, constituyen el 'Síndrome Climatérico' que es común al 80\% de las mujeres. El 20\% restante no sufre cambios significativos" (2007: 1).

Otro aspecto analizado fueron los mitos y estereotipos que los y las encuestadas asumen, como hechos asociados al lapso que atraviesan, lo cual puede ser determinante para enfrentar esta etapa, así como, afectar la vivencia de su sexualidad. Estas creencias erróneas que la sociedad ha implantado en las personas, como la de asociar esta etapa con eventos que limitan las capacidades físicas, fisiológicas y emocionales, hacen que se conciba como una enfermedad, la cual debe ser tratada (Organización Panamericana de la Salud, 2000). Estas concepciones mencionadas anteriormente, no solo dañan la integridad social de la mujer, sino también la vivencia de esta etapa en el hombre, la cual se ve perjudicada por la existencia de mitos, en parte, relacionados con factores como: el machismo, el cual les limita lo que están viviendo en esta etapa, sus inquietudes $y$ dudas, haciendo que estos mitos perduren $y$ se incrementen. Por esta razón, debido a estos conceptos erróneos de virilidad que se posee, la enseñanza se convierte en un método indispensable para aclarar estos mitos y tabúes presentes en la actualidad (Rodríguez, 2009).

Cabe destacar que, si bien es cierto, se ha dado una mayor apertura al trato de la temática de la menopausia y la andropausia en los programas de educación para la salud que se brindan a la comunidad costarricense, la población continúa manejando conceptos erróneos, conceptualizando la menopausia únicamente como una disminución de las hormonas femeninas y la andropausia como un proceso masculino. Evidencia de esto son los resultados que arroja el presente estudio, mostrando la poca claridad que poseen los y las encuestadas sobre ambos conceptos.

Por otra parte, en cuanto a las edades que abarca esta etapa, se pudo apreciar que en su gran mayoría, se tiene claro cuál es el intervalo de edad correspondiente a este período, contribuyendo a la disminución de la ansiedad que puede generar dichos cambios. Es importante resaltar que aun cuando no existe una edad determinada para su inicio, debido a que los organismos femeninos son diferentes $y$ por tanto, dicho lapso puede variar, se considera que este proceso puede desenvolverse entre los 35 a 65 años, período en que se desarrollan las etapas de transición: perimenopausia y menopausia (Bonifacio et ál., 2007). 
Respecto al concepto de andropausia, se ve evidenciado que la mayoría de los encuestados no saben qué es o manejan un concepto erróneo sobre el mismo. Esto puede deberse a la desinformación sobre este período; una muestra de esto es el desarrollo reciente de investigaciones $y$ estudios relacionados con la masculinidad, que dejan de lado temas allegados de interés para la población masculina, como lo son el climaterio masculino y la andropausia; aunado a esto se encuentran las políticas de salud, las cuales carecen de programas que incorporen a la población adulta masculina (Arrondo, Cuesta, Grasa, Ponz, Solchaga, Aldave, Pascual, Napal e Ipiens, 2004).

Dado los hallazgos obtenidos, algunos autores mencionan que el climaterio y la andropausia vienen siendo una definición parecida; sin embargo, el climaterio se refiere a los cambios que sufren tanto los hombres como las mujeres, mientras que la andropausia se refiere a los “(...) cambios hormonales que tienen lugar en los hombres de edad media relacionados con el proceso de envejecimiento, de manera similar a lo que ocurre con la menopausia en las mujeres..." (Silva, 2006: 1). Como lo señala el autor, estos cambios son provocados por una disminución de las hormonas masculinas, incitando una serie de cambios físicos $y$ psicológicos.

En cuanto a la edad en que se desarrolla la andropausia, esta se da entre los 40 y 60 años, puesto que la edad promedio de este proceso, se observa en hombres mayores de 45 años; no obstante, se sabe que a partir de la tercera y hasta la cuarta/quinta década de vida, se inicia la declinación de los niveles sanguíneos de andrógenos endógenos, aunque sin cambios detectables (Silva, 2006).

Por su parte, otra de las temáticas analizadas, es la referente a la sexualidad, la cual fue estudiada desde cada uno de sus componentes, como lo son: las relaciones interpersonales, proyecto de vida, sexo, género, erotismo y espiritualidad.

En lo referente al proyecto de vida de los y las participantes, como se mostró en el apartado anterior, un gran porcentaje no tiene ningún proyecto atinente al plan de vida que se orienta a saber qué es lo que quiere cada persona, en función de sus intereses, competencias, habilidades, formación, así como, fomentar el aprecio y el respeto que poseen por sí mismos (Masso, 2009).

Aunque el porcentaje de encuestados que no tenía claro un proyecto de vida, es mucho menor al que sí lo tenía, es importante rescatar este elemento, puesto que un proyecto de vida bien establecido en cada una de las etapas de vida, es fundamental para todos los individuos como parte de la construcción de su sexualidad; ya que esto mismo incentiva motivos suficientes para luchar y seguir adelante, asimismo, es pieza clave en la construcción del sentido de identidad, ayudando a fomentar la realización y satisfacción personal.

Además es importante destacar que la ilusión para alcanzar las metas es, muchas veces, el motor que empuja la vida de las personas a establecer proyectos a futuro que les permita planear estrategias y prever los obstáculos potenciales que puedan limitar o entorpecer el logro de su proyecto de vida (Domínguez, 2007).

En cuanto a la capacidad de relacionarse con los demás, se considera que para la vivencia plena de la vida, es importante tener una adecuada relación interpersonal donde exista una comunicación recíproca entre dos o más personas, en la cual se involucren los aspectos de la habilidad para comunicarse: el escuchar, la solución de conflictos y la expresión auténtica de cada quién (Buitrago y Amaya, 2001). Además, es fundamental comprender que los seres humanos viven en constante relación con otras personas; somos una especie que está en continuo contacto con los demás y necesitamos de afecto, lo que convierte a esta interacción en una necesidad básica para vivir; en otras palabras, se necesita de los demás para sobrevivir (Aldana, Gómez, Sánchez y Gaviño, 2007).

Basándose en los resultados anteriores, se afirma que durante el proceso de las relaciones interpersonales se requiere del desarrollo de destrezas, las cuales se van adquiriendo y moldeando, según cada etapa de vida en la que se encuentran; asimismo, realizando cambios en la personalidad $y / 0$ autoestima de cada persona. Aunado a lo anterior, el mantenimiento 
de relaciones interpersonales eficaces, también dan un sentido de pertenencia, lo cual es un elemento trascendental en la construcción y el desarrollo de la identidad personal, ya que fortalece la seguridad de las mismas (Buitrago y Amaya, 2001).

Es relevante indagar cuatro componentes básicos de la sexualidad: sexo, género, espiritualidad y erotismo. En primer lugar, es transcendental determinar el concepto que manejaban los y las encuestadas sobre sexo, por cuanto existe una gran cantidad de definiciones; no obstante, en este trabajo el concepto de sexo debe entenderse como el "conjunto de características biológicas que definen al espectro de humanos como hembras y machos", lo cual es congruente con la definición de la Organización Mundial de la Salud (2000). Un número representativo de los encuestados poseía un concepto equivocado sobre esa definición, lo cual muestra una gran carencia de información sobre este tema. El reconocer esto es fundamental ya que es necesario llenar estos vacíos, con el fin de que las personas logren diferenciar la variedad de componentes que encierra la sexualidad de las personas, lo que ayudará a una vivencia más plena de la misma.

Asociado a este concepto, otro de los tópicos analizado fue la concepción de género, el cual se tiende a confundir $y$ asemejar con el concepto de sexo, por esto se indagó en la definición que poseían los y las participantes, ya que muchas veces suelen usarse como sinónimos, aunque sean conceptos diferentes. En este caso, solamente un grupo pequeño (27\%) acertó el componente como "conjunto de características sociales y culturales asignadas a las personas en función de su sexo" (Instituto de las Mujeres de la Ciudad de México, 2009). La influencia que posee este elemento en el desarrollo de la sexualidad es fundamental, pues se encuentra presente en las personas desde la niñez, debido a que al intervenir toda la sociedad en el proceso de aprendizaje, desde la familia hasta la comunidad, en general, se van dando pautas, por medio de una serie de simbolismos, de cómo se deben comportar las mujeres y los hombres, para que encajen en ella. Como lo apuntan Valdez, Gysling y Benavente (1999):
La conducta sexual de los hombres y las mujeres y sus resultados están determinados y condicionados por las características familiares, el concepto de los "roles sexuales", experiencias anteriores y oportunidades, más que por el hecho de ofrecer información o instrucción sexual (1999: 1).

Asimismo, se destacaron los roles asignados para cada uno de los géneros, los cuales son muy característicos de cada sociedad; sin embargo, se mantienen estándares universales que se deben respetar. Los roles están presentes en la vida cotidiana, ya que son la razón de ser, sentir y actuar de cada persona. Estas acciones responden a las exigencias que se les piden por ser mujer u hombre, además, dictan la manera de relacionarse entre los dos sexos (King, 1984).

Comprender la definición de cada concepto es útil para las personas, ya que les permite tener claro los roles sociales específicos para la mujer y el hombre, permitiendo generar un cambio en el pensamiento y el comportamiento, debido a que se logra comprender que estas exigencias sociales inherentes al género, son solo frutos de una sociedad patriarcal, pensamiento que en el trascurso de la socialización primaria $y$ secundaria, se les ha inculcado a las personas.

Otro componente que se investigó como parte del componente de la sexualidad, fue el erotismo. Alrededor de este tema son muchas las ideas equivocadas que se tienen al respecto, lo cual se apoya con la evidencia en los resultados. Este concepto debe entenderse como: "los procesos humanos entorno al apetito por la excitación sexual, la excitación misma y el orgasmo, sus resultantes en la calidad placentera de esas vivencias humanas, así como las construcciones mentales alrededor de estas experiencias" (Rubio, 2009).

$\mathrm{El}$ erotismo se debe comprender como el medio, por el cual se puede adquirir un conocimiento profundo sobre el cuerpo y los deseos de sí mismo, además de los del otro; es por esto que el verdadero erotismo se expresa cuando cada uno trata de comprender al otro, hace lo que le gusta y a la vez, está haciendo lo que le gusta al otro (Martínez, 2008). Aunado a lo 
anterior, permite percibir las sensaciones de nuestro cuerpo, solos o con los demás, proporcionándo placer y satisfacción, lo cual constituye el objetivo principal.

Como último componente de la sexualidad, se analizó el tema de la espiritualidad; para ello se indagó en el concepto que poseían las personas respecto a este, donde el más evidente fue: "conjunto de aspiraciones, convicciones, valores $y$ creencias que permiten a cada persona orientar sus proyectos de vida" (Campos, 2008); sin embargo, también la consideraron como un componente exclusivo de una religión, confundiendo, de esta manera, la espiritualidad con la religión, lo cual es uno de los problemas más frecuentes en la sociedad. Es debido a esto que, muchas veces, las personas se restringen en la vivencia de la espiritualidad, ya que consideran que esta no se encuentra en ellos, al no pertenecer a ninguna religión. Por lo tanto, es importante que las personas comprendan que una construcción propia de cada ser humano puede variar en el transcurso de la vida y que cada persona la expresa de manera diferente. La espiritualidad permite creer en sí mismo; es a través de ella que las personas se encuentran consigo mismo(a) y fortalecen su yo interno, reforzando en un marco de valores, que forman un todo aquello que se es, se cree y se actúa.

La espiritualidad no puede ser entendida sola, sino como un componente más de la sexualidad y por tanto, al establecer cualquier intervención con la persona adulta mayor, se debe tratar integralmente la sexualidad, partiendo del cambio de visión de la genitalización de la etapa de la andropausia y la menopausia, hacia una que conceptualice la sexualidad integralmente, comprendiendo que está compuesta de una serie de dimensiones que deben ser fortalecidas tendientes a "encontrándose bien con su interior".

\section{CONSIDERACIONES FINALES}

$\diamond \quad$ Los resultados del estudio ponen en evidencia que las personas que se encuentran en la etapa de adultez intermedia poseen vacíos de información con respecto al proceso de vida que atraviesan, lo que afecta de manera negativa la vivencia plena durante la misma, como por ejemplo en su sexualidad, debido a que el desconocimiento en el tema reduce las posibilidades de actuar y/o enfrentar la realidad de los cambios que se presentan.

$\diamond \quad$ Entre los cambios más comunes de esta etapa que han atravesado los entrevistados se encuentran: trastornos del sueño, resequedad vaginal, disminución del deseo sexual, alargamiento del tiempo para conseguir una segunda erección, afecciones cardiovasculares, adelgazamiento de las paredes vaginales, entre otras.

$\diamond \quad$ La educación en sexualidad es escasa para los periodos de perimenopausia, menopausia, climaterio femenino y andropausia; es por esto que la educación para la salud es una estrategia fundamental en el área de promoción, con la cual se deben de llenar los vacíos de información que las personas poseen, cambiando la perspectiva con respecto a esta etapa del desarrollo; cabe resaltar que se propone como estrategia educativa la andragogía.

$\diamond \quad$ Conocer los cambios que se pueden generar en esta etapa de vida, es una herramienta que contribuye a ayudar a que las personas que atraviesan esta etapa del desarrollo disminuyan el miedo, la ansiedad y las dudas que surgen al desconocer qué es lo que esta pasando, lo cual se logra como se mencionó en la consideración anterior por medio de un proceso educativo.

$\diamond \quad$ La autoestima se ve afectada debido a la falta de información y los perjuicios sociales que se dan en torno a la etapa de vida que atraviesan. Como se evidenció en el estudio, este es un tema fundamental a la hora de abordar la temática sobre la sexualidad en los períodos de perimenopausia, menopausia, climaterio femenino y andropausia, debido a que el amor propio es el eslabón base del cual se parte para lograr un cambio en la perspectiva de los individuos. Por tanto, todo proceso educativo que se realice con este grupo población debe tener de base el manejo de la autoestima. 


\section{RECOMENDACIONES}

$\diamond \quad$ Es necesario llenar los vacíos de información que poseen las personas que se encuentran en los períodos de perimenopausia, menopausia, climaterio femenino $y$ andropausia con respecto a los cambios que se producen en estas etapas de vida con el fin de lograr una vivencia plena de su sexualidad.

$\diamond \quad$ Con la finalidad de que la familia conozca los procesos del ciclo vital que se atraviesan en la adultez intermedia, es indispensable realizar estrategias de educación para la misma, debido a que, los y las participantes refirieron la importancia de comprensión y apoyo por parte de su núcleo familiar.

$\diamond \quad$ Promover la creación de grupos de apoyo de personas que se encuentren atravesando la misma etapa de desarrollo, donde puedan compartir sus experiencias, temores, dudas $y$ adquirir herramientas para afrontar juntos los diferentes cambios.

$\diamond \quad$ Incluir en las ferias de la salud, información oportuna $y$ veraz sobre los procesos de perimenopausia, menopausia, climaterio femenino y andropausia, así como, los distintos tratamientos para el manejo de los síntomas.

BIBLIOGRAFÍA

Aldana, E.; Gómez, M.; Sánchez, M. y Gaviño, F. "Áreas de conflicto en mujeres en la etapa del climaterio en dos grupos de psicoterapia”. Revista de Ginecología y Obstetricia de México 75. 2007: 332-340.

Araya, A.; Urrutia, M. y Cabieses, B. "Climaterio y postmenopausia: aspectos educativos a considerar según la etapa del periodo". Revista Ciencia y Enfermería XII (1). 2006: 19-27.

Arrondo, J.; Cuesta, J.; Grasa, M.; Ponz, A.; Solchaga, J.; Aldave, I.; Pascual, S.; Napal, S. e Ipiens, A. "Andropausia: ¿un síndrome que se debe tratar?". Revista Internacional de Andrología 2 (2). España, 2004: 60-67.
Barrantes, R. Investigación: un camino al conocimiento, un enfoque cuantitativo y cualitativo. San José, Costa Rica: Editorial EUNED, 2006.

Benítez, J. Climaterio. 2008. En: <http://www. semergen.es/semergen/contentFiles /13666/es/climaterio.pdf> [consultado el 17 de octubre de 2010].

Bonifacio, C.; Espínola, L.; Lavado, F.; Reguera, O. y Varas, P. Influencia del entorno familiar y laboral en relación a la autoestima de la mujer climatérica en el Hospital I Luís Albrecht. Es Salud de Trujillo, noviembre 2007. En: <http://www.scribd.com/doc/902972/ el-climaterios [consultado el $17 \mathrm{de}$ octubre de 2010].

Buitrago, O. y Amaya, B. "Educación Personalizada, una modalidad educativa”. Revista de Ciencias Humanas 26. Colombia, 2001. En: <http://www. utp.edu.co/ chumanas/revistas/revistas/ rev26/buitrago.htm>

Campos, C. Ética y religión, propuestas para una relación saludable. La Mancha: Ediciones de la Universidad de CastillaLa Mancha, 2008.

Chacín, M. y Briceño, M. Cómo generar líneas de investigación: sugerencias prácticas para profesores $y$ estudiantes. Segunda edición. Venezuela: Publicaciones del Decanato de Postgrado, Universidad Nacional Experimental Simón Rodríguez, 2001.

Clavijo, L. "Evaluación de funciones familiares en pacientes con Síndrome Climatérico en el Hospital Obrero Nro. 2 de la Caja Nacional de Salud Cochabamba-2008". Rev. Med. (Cochabamba) 20 (30). Cochabamba, 2009.

Domínguez, L. "Proyecto de vida y valores: condiciones de la personalidad madura y saludable". Boletín Electrónico de la Asociación Oaxaqueña de Psicología 3 (1). 2007: 44-58. En: <www.conductitlan. net/40proyecto_de_vida.pdf $>$ [consultado el 17 de octubre de 2010].

Guzmán, J., Huenchuan, S y Montes de Oca, V. "Marco teórico conceptual sobre redes de 
apoyo social de las personas mayores". CEPAL-SERIE Seminarios y conferencias 30. Santiago, Chile. Publicaciones Naciones Unidas, 2003: 23-32. En: $<$ http://www.eclac.cl/publicaciones/ xml/2/14182/lcl1995_1.pdf> [consultado el 16 octubre de 2011 ].

Instituto de las Mujeres de la Ciudad de México. ¿Qué es género? 2009. En: $<w w w . i n m u j e r . d f . g o b . m x / g e n e r o / p d f /$ generoquees.pdf $>$ [consultado el 17 de octubre de 2010].

King, I. Enfermería como profesión. Filosofía, principios y objetivos. México: Editorial LIMUSA, 1984.

Martínez, V. El Erotismo. 2008. En: <www. sexuar.com.ar/divulgacion/ erotismo. html> [consultado el 17 de octubre de 2010].

Masso, F. Autoestima. 2009. En: <www.es.salut. conecta.it/pdf/injuve/ IIguianorexia_4. pdf $>$ [consultado el 17 de octubre de 2010].

Medina, C. "Andropausia y menopausia". Revista de la Facultad de Ciencias Médicas. Enero-junio 2008: 7-8. En: $<$ http://www.bvs.hn/RFCM/pdf/2008/pdf/ RFCMVol5-1-2008-2.pdf> [consultado el 17 de octubre de 2010].

Moynihan, Clare. "Theories of masculinity". BMJ 317 (7165). 1998: 1072-1075.

Organización Mundial de la Salud-oms. "Investigaciones sobre menopausia en los años noventa. Informe de un grupo científico de la OMS". Serie de Informes Técnicos 866. Ginebra, Suiza. 1996.

Organización Mundial de la Salud-oms. "Promoción de la salud sexual: recomendaciones para la acción". 2000. En: <www.perueduca.edu.pe/ adolescentes-y-salud/.../sxsaludsexualops. pdf $>$ [consultado el 17 de octubre de 2010].

Organización Mundial de la Salud-oms. "Autoevaluaciones de la salud en la Encuesta Mundial de Salud 2002: correlación con el nivel educativo". Boletín de la Organización Mundial de la Salud 88. Febrero 2010: 81-169.
Organización Panamericana de la Salud (OPS). Promoción de la salud sexual. Recomendaciones para la acción. Washington DC: OPS, 2000.

Organización Panamericana de la SaludOrganización Mundial de la Salud. Promoción de la salud sexual. 2000. En: $<$ http://www.infoabu.com/Salud-SexualPromocion-PAHO-OMS.pdf> [consultado el 25 de octubre de 2010].

Programa Estado de la Nación. Decimoquinto Informe Estado de la Nación en Desarrollo Humano Sostenible. San José, Costa Rica: Programa Estado de la Nación, 2009.

Rodríguez, B. "Climaterio femenino". Revista Científica de UCES VIII (2). 2009. En: <http://desarrollo.uces.edu.ar:8180/ dspace/bitstream/123456789/734/1/ Climaterio_masculino.pdf> [consultado el 17 de octubre de 2010]

Rubio, E. Modelo Holónico de la sexualidad humana. 2009 . En: <www. formacion-integral.com.ar/index 2 . php?option=com_content $>$ [consultado el 17 de octubre de 2010].

Sánchez, R.; Honrubia, M. y Chacón, M. Guía básica de educación afectivo-sexual para personas con discapacidad visual. España: Publicacions I edicions de la Universitat de Barcelona, 2005.

Silva, J. "Andropausia. Estado actual y conceptos básicos". Rev. Universitas Médica 47 (1). Bogotá, Colombia. Pontificia Universidad Javeriana, 2006: 17-23. En: <http://www. redalyc.org/src/inicio/ArtPdfRed. jsp?iCve $=231018678003>$

Valdés, T.; Gysling, J. y Benavente, M. El poder de la pareja, la sexualidad y la reproducción. Mujeres de Santiago, Chile. 1999.

Fecha de ingreso: $22 / 02 / 2012$ Fecha de aprobación: 08/08/2012 
